\title{
KAHBPS-3
}

\section{Development of platform for education and learning in minimally invasive pancreatic surgery}

\author{
Sung Hoon $\mathrm{CHOI}^{*}$
}

Division of Hepatobiliary and Pancreas, Department of Surgery, CHA Bundang Medical Center, Seongnam, Korea

Lecture: In an era when minimally invasive pancreatic surgery is rapidly increasing, there is a desperate need for an educational program for the safe establishment and standardization of minimally invasive pancreaticoduodenectomy. This study aimed to develop a program that will enable easy access to educational materials and software development that can perform surgical simulation as an educational platform for minimally invasive pancreaticoduodenectomy.

Two main training programsare development of surgical simulator and video-based training program. Surgical simulator is an in vitro mastery-based training kit which can practice two main reconstruction procedures of hepaticojejunostomy and pancreaticojejunostomy. Another is a video-based training program building webhard, which was categorized as 1 . Korean minimally invasive pancreatic surgery (K-MIPS) education program, 2. Technical notes, and 3. K-MIPS online mentoring program. The K-MIPS education program consisted with expert's videos and public videos, allowing novice surgeons to learn the techniques of experienced surgeons. Technical notes provide lecture materials for members to upgrade their theoretical knowledge. K-MIPS online mentoring program is a highlight of this education program. This program contains three stages. Stage 1 is a video library, which provides important surgical pitfalls in procedure segmentation for stepwise approach. Here, novice surgeons can learn the each procedure in step-by-step following the experts' surgery. Stage 2 and 3 are online mentoring system. Novice surgeons can upload their in-vitro and in-vivo experience. Then, members of the board of education of K-MIPS plan to provide evaluation and hearfelt mentoring.

With this online educational platform, we hope to establish feasible, practical, and effective surgical educational system for safe implementation of minimally invasive pancreatectomy in our society. 